\title{
STUDIES OF TRANSVERSE SPIN EFFECTS AT JLAB
}

\author{
H. AVAKIAN, P. BOSTED, V. BURKERT, L. ELOUADRHIRI FOR THE \\ CLAS COLLABORATION \\ Jefferson Lab, Newport News, VA 23606, USA
}

\begin{abstract}
We present ongoing and future studies of single-spin asymmetries in semi-inclusive electroproduction of pions using the CEBAF polarized electron beam. Kinematic dependences of single-spin asymmetries have been measured in a wide kinematic range at CLAS with a polarized $\mathrm{NH}_{3}$ target. Significant target-spin $\sin 2 \phi$ and $\sin \phi$ asymmetries have been observed, indicating a non-zero Collins fragmentation function and supporting future SIDIS measurements with upgraded JLab.
\end{abstract}

\section{Factorization in Semi-Inclusive DIS}

In recent years it has become clear that appropriate exclusive and semiinclusive scattering processes may provide access to parton distributions, generalized to account for not only longitudinal but also transverse degrees of freedom of partons ${ }^{1-16}$. SIDIS cross section at leading twist has eight contributions related to different combinations of polarization states of the incoming lepton and the target nucleon ${ }^{17}$. Corresponding structure functions factorize into transverse momentum dependent (TMD) parton distribution and fragmentation functions (FF), and soft and hard parts ${ }^{17}$. It was shown, that the hard factors in the SIDIS cross section for different contributions are similar at one-loop order ${ }^{17}$ and may cancel to large extend in asymmetry observables.

Single-spin asymmetries (SSA) in the distribution of lepto-produced hadrons in the azimuthal angle around the virtual photon direction are a valuable tool for the exploration of transverse spin and momentum degrees of freedom in nucleon structure. A key goal of Semi-Inclusive DIS (SIDIS) studies at moderate energies accessible at JLab is to carefully study the transition between the nonperturbative and perturbative regimes of QCD using simultaneous measurements of the $Q^{2}$ and $x$ dependences of cross sections and beam/target spin asymmetries for different final state hadrons and extraction of the corresponding structure functions and separation of the contributions of different distribution and fragmentation functions. 
A major issue in studies of semi-inclusive scattering at moderate beam energies is the separation of contributions from current fragmentation (active parton) and target fragmentation (spectators). Systematic studies of factorization of $x, z$ and $P_{h \perp}$ dependences for spin-dependent observables and for different pion flavors will be required to do a flavor decomposition of various PDFs from measured spin and azimuthal asymmetries in SIDIS. It was shown that the CLAS data ${ }^{18,19}$ already at existing energies are consistent with factorization and suggest that the high energy-description of the SIDIS process can be extended to the moderate energies of the CLAS measurements.

\section{Single-Spin Asymmetries}

Large SSAs, observed in hadronic reactions for decades ${ }^{20,21}$ have been among the most difficult phenomena to understand from first principles in QCD. Recently, SSA measurements were reported in semi-inclusive DIS (SIDIS) by the HERMES collaboration at HERA ${ }^{22,23,24}$ for longitudinally and transversely polarized targets, by the COMPASS collaboration with a transversely polarized target ${ }^{25}$ and by the CLAS collaboration at JLab with a polarized beam and longitudinally polarized target ${ }^{18,19}$. In general, such single-spin asymmetries require a correlation of a particle spin direction and the orientation of the production or scattering plane. In hadronic processes, such correlations can provide a window into the physics of initial and final state interactions at the parton level ${ }^{8}$.

\subsection{Leading order SSA}

For polarized targets, several azimuthal asymmetries already arise at leading order. The following contributions were investigated in Refs. $3,5,8,11,28,29$ :

$$
\begin{aligned}
\sigma_{U L}^{\sin 2 \phi} & \propto S_{L} 2(1-y) \sin 2 \phi \sum_{q, \bar{q}} e_{q}^{2} x h_{1 L}^{\perp q}(x) H_{1}^{\perp q}(z), \\
\sigma_{U T}^{\sin \phi} & \propto S_{T}(1-y) \sin \left(\phi-\phi_{S}\right) \sum_{q, \bar{q}} e_{q}^{2} x h_{1}(x) H_{1}^{\perp q}(z), \\
& +S_{T}\left(1-y+y^{2} / 2\right) \sin \left(\phi+\phi_{S}\right) \sum_{q, \bar{q}} e_{q}^{2} x f_{1 T}^{\perp q}(x) D_{1}^{q}(z),
\end{aligned}
$$

The $\phi$ is the azimuthal angle between the scattering plane formed by the initial and final momenta of the electron and the production plane formed

by the transverse momentum of the observed hadron and the virtual photon, 
$\phi_{S}$ is the azimuthal angle of the transverse spin in the scattering plane, $S_{L}$ and $S_{T}$ are longitudinal and transverse polarizations with respect to virtual photon direction.

The spin-dependent moments $(\sin \phi, \sin 2 \phi)$ of the semi-inclusive cross section can be extracted in a fit to the normalized-yield asymmetry

$$
A_{U L}(\phi)=\frac{1}{P_{T}} \frac{N^{+}-N^{-}}{N^{+}+N^{-}}
$$

Here $N^{ \pm}$is the number of events for target polarizations antiparallel/parallel to the incoming beam direction, $P_{T}$ is the target polarization,

The $\sin \phi$ moment of the SIDIS cross section with a transversely polarized target $\left(\sigma_{U T}\right)^{32}$ contains contributions both from the Sivers effect (T-odd distribution) ${ }^{4}$ and the Collins effect (T-odd fragmentation) ${ }^{3}$. Contributions to transverse SSAs from T-odd distributions of initial quarks ( $f_{1 T}^{\perp q}(x)$ term) and T-odd fragmentation of final quarks $\left(H_{1}^{\perp q}(z)\right.$ term) could be separated by their different azimuthal dependence (see Eq.2).

The HERMES Collaboration has recently measured a transverse spin asymmetry in SIDIS providing the cleanest evidence to date for the existence of a non-zero Collins function ${ }^{24}$, which describes the fragmentation of a transversely polarized quark into pions. This finding is supported by the preliminary data from BELLE ${ }^{26}$ indicating a non-zero Collins effect. The large target SSA in semi-inclusive pion production measured at CLAS and analyzed in terms of the Collins fragmentation ${ }^{27}$, also indicate a significant Collins function. To reveal the source of SSA and accomplish the separation of Collins and Sivers contributions, measurements with different target polarizations and with detection of different final state particles may be required.

One important, unique feature of Collins mechanism is the presence of a leading twist $\sin 2 \phi$ dependent SSA, so-called "Kotzinian-Mulders asymmetry" ${ }^{28}$, the only azimuthal asymmetry arising for the longitudinally polarized target in leading order ${ }^{5,28,29}$ (see Eq. 1). This contribution involves the TMD Collins fragmentation function $H_{1}^{\perp}{ }^{3}$ and the Mulders distribution function $h_{1 L}^{\perp}{ }^{1,5}$, describing the transverse polarization of quarks in a longitudinally polarized proton ${ }^{5,28,29}$. The distribution function $h_{1 L}^{\perp}$ is also accessible in double polarized Drell-Yan, where it gives rise to a $\cos 2 \phi$ azimuthal moment in the cross section ${ }^{30}$.

Measurements of the $\sin 2 \phi$ SSA allow the study of the Collins effect with no contamination from other mechanisms. A recent measurement of $\sin 2 \phi$ moment of $\sigma_{U L}$ by HERMES ${ }^{22}$ is consistent with zero. A measurably 


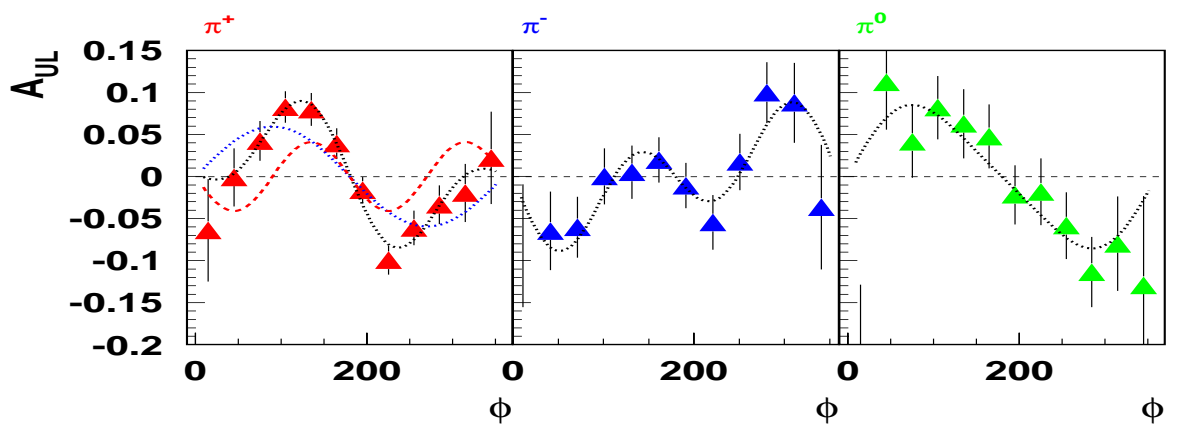

Figure 1. Preliminary target SSA as a function of azimuthal angle $\phi$ from data at $5.7 \mathrm{GeV}$. Dashed and dotted lines correspond to $\sin 2 \phi$ and $\sin \phi$ moments respectively

large asymmetry has been predicted only at large $x(x>0.2)$, a region wellcovered by JLab ${ }^{31}$. The data for $\pi^{+}$(Fig. 1) show a clear $\sin \phi$ and $\sin 2 \phi$ modulations from which a $\sin \phi$ moment of $0.058 \pm 0.011$ (stat) and $\sin 2 \phi$ moment of $-0.041 \pm 0.011$ (stat) have been determined.

Future measurements approved for longitudinal target with a $6 \mathrm{GeV}$ beam will significantly improve the quality of this measurement (see Fig. 2). Upgrade of JLab, enabling measurements at $11 \mathrm{GeV}$ and at much higher luminosity, will help to pin down the corresponding transverse momentum distribution functions.

Projections for transverse target single-spin asymmetry measurements with CLAS at $11 \mathrm{GeV}$ are plotted in Figs. 3,4. The curves are calculated by Efremov et al. for $z>0.45$ and the missing mass of the $e^{\prime} \pi$ system $\left(M_{X}\left(\pi^{+}\right)>1.1 \mathrm{GeV}\right)$. The asymmetry is integrated over all hadron transverse momenta. The extraction of the transversity from $A_{U T}^{\sin \phi}$ could be performed using parameterizations for the unpolarized distribution functions $u(x)$ and $\bar{d}(x)$ and certain approximations for the polarized Collins fragmentation function $H_{1}^{\perp}$.

The same measurement of the transverse asymmetry, provides also access to the Sivers effect. Measurements with proton and neutron targets will enable direct extraction of information on flavor partners of the Sivers function, as the extraction of the Sivers distribution function doesn't require additional information on the polarized quark (Collins) fragmentation functions. Furthermore, using transverse target asymmetries for $\pi^{0}$ would allow 


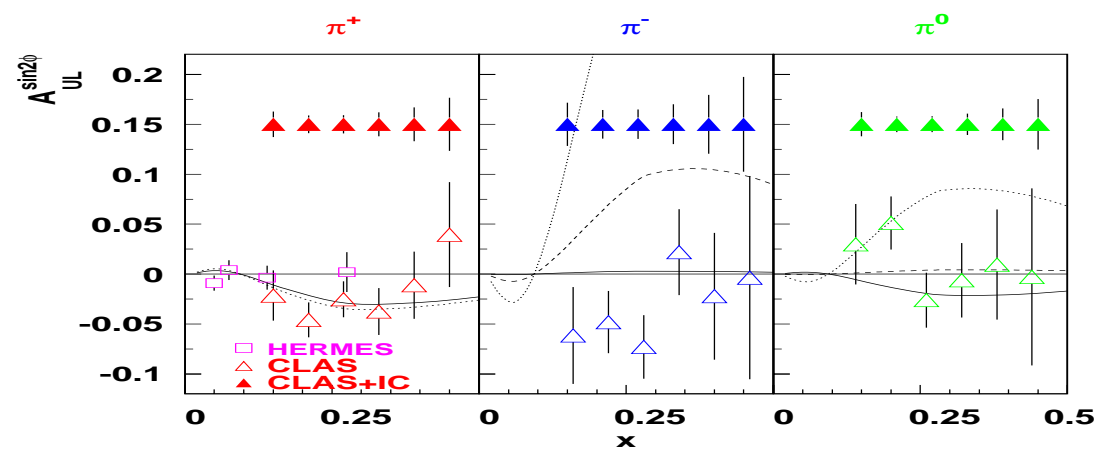

Figure 2. Dependence on $x$ of longitudinally polarized target SSA, $A_{U L}^{\sin 2 \phi}$. Squares are HERMES data for $A_{U L}^{\sin 2 \phi}$, open triangles are CLAS measurements with $\mathrm{NH}_{3}$ target and triangles represent expected statistical errors from JLAB E05-113 proposal at 6 $\mathrm{GeV}$ with 1000 hours of data taking. The curves are the theory prediction based on the Collins mechanism ${ }^{31}$ for different ratios of $H_{u n f a v} / H_{\text {fav }}$. The solid, dashed and dotted lines are respectively for $0,-1.1$, and -5 .

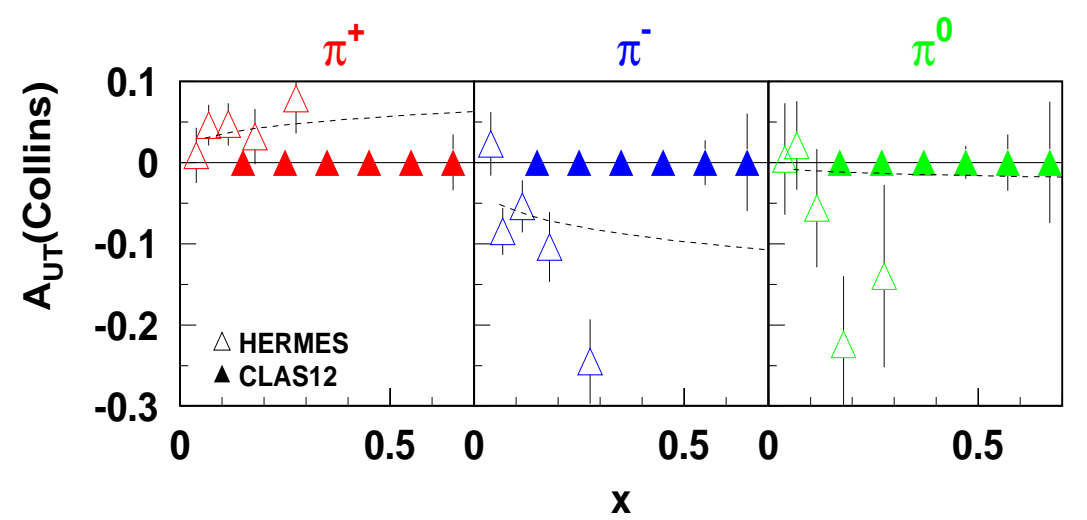

Figure 3. Projected transverse spin asymmetry from the Collins effect $\left(A_{U T}^{\sin \left(\phi+\phi_{S}\right)}\right)$ in single $\pi$ production with CLAS at $11 \mathrm{GeV}$. The projected error bars have been calculated assuming a luminosity of $10^{35} \mathrm{~cm}^{-2} \mathrm{~s}^{-1}$, with a $\mathrm{NH}_{3}$ target polarization of $85 \%$ and a dilution factor 0.176 , and 2000 hours of data taking.

extraction of Sivers function even without any knowledge of the unpolarized fragmentation functions providing a model independent way for Sivers 
function studies. The $\pi^{0}$ studies will also have an important advantage of being less affected by exclusive vector meson background. Projections for transverse asymmetry measurements with upgraded CLAS and transverse target and also for extraction of the Sivers distribution function are shown in Fig. 4.
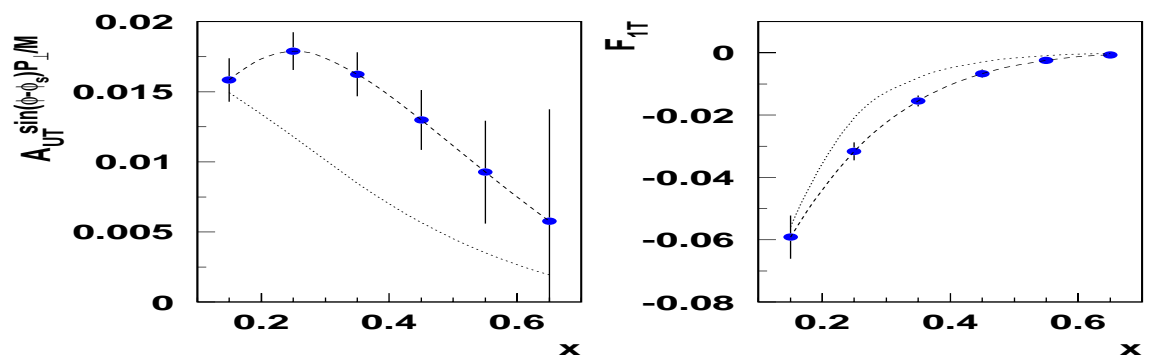

Figure 4. Projected transverse spin asymmetry from the Sivers effect $\left(A_{U T}^{\sin \left(\phi-\phi_{S}\right)}\right)$ in single $\pi$ production with CLAS at $11 \mathrm{GeV} . F_{1 T}$ is the sum of Sivers distributions of $u$ and $d$ quarks weighted by their charges squared. The curves are theory predictions for two models of the Sivers function ${ }^{31}$.

\subsection{Sub-leading order $S S A$}

With better experimental accuracy it may be possible to isolate the highertwist effects in hard processes, which arise from the quantum mechanical interference of partons in the interacting hadrons. The higher-twist terms are important for understanding long-range quark-gluon dynamics and may be accessible through measurements of certain asymmetries ${ }^{2, ?, 29,33}$, where they appear as leading contributions.

The complete analysis up to sub-leading-twist and leading order in $\alpha_{s}$ of longitudinal single-spin asymmetries in semi-inclusive DIS was presented in Ref. ${ }^{34}$, completing previous work of Refs. ${ }^{5,32}$. All contributions involve either combinations of sub-leading-twist distribution functions $\left(e, h_{L}, g^{\perp}\right.$, $f_{L}$ ) with leading-twist fragmentation functions or of leading-twist distribution functions in conjunction with sub-leading-twist fragmentation functions $\left(G^{\perp}, E\right)^{14,34,35}$.

Distribution functions $g^{\perp}$ and $f_{L}{ }^{14,34}$ are odd under time reversal (Todd) and don't have a simple probabilistic interpretation. Their contributions are similar to the leading order Sivers effect, so the same extraction 
procedure can be used as in case of Sivers functions from measurements of SSAs for $\pi^{0}$ s (see Fig. 4). In general both SSAs contain additional contributions depending on the fragmentation function $G^{\perp}$, which is at present unknown. This makes beam and target single-spin asymmetry measurements complimentary and global analysis is required to separate different contributions.

So far factorization has been proven only for leading-twist observables in semi-inclusive deep-inelastic scattering with hadrons in the current fragmentation region. A factorization proof for sub-leading-twist observables is still open. At the moment there are only few model predictions ${ }^{14,31}$ and no firm experimental information about any of the sub-leading-twist terms exist.

Measurements of $A_{U L}$ and $A_{L U}$ reported by HERMES ${ }^{22,23,37}$ and CLAS ${ }^{18,19}$ are first indications about the size of such sub-leading-twist effects in azimuthal target-spin and beam-spin asymmetries (see Fig. 5).
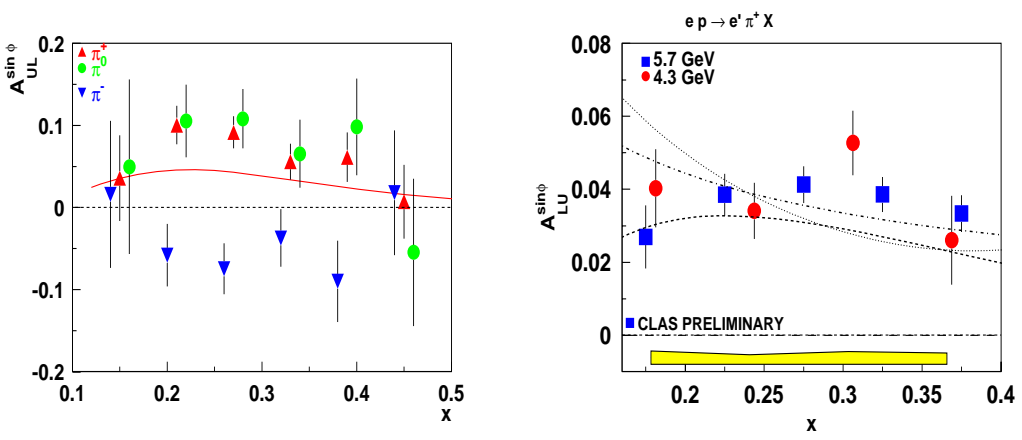

Figure 5. Sub-leading SSA with polarized target (left) or beam (right). The sin $\phi$ moment of target SSA for $5.7 \mathrm{GeV}$ beam extracted for $\pi^{+}, \pi^{-}$, and $\pi^{0}$. The beam-spin azimuthal asymmetry $(\sin \phi$ moment of the cross section) extracted from hydrogen data at $5.7 \mathrm{GeV}$ (squares) and $4.3 \mathrm{GeV}$ (circles) as a function of $x_{B}$ in a range $0.5<z<0.8$. Curves represent calculations performed assuming only the Sivers effect (dash-dotted lines ${ }^{35}$, dotted ${ }^{36}$ ) or only the Collins effect (dashed line) ${ }^{31}$ contributes to $A_{L U}$.

\section{Conclusions}

An experimental investigation of properties of 3D PDFs, requiring precision measurements at upgraded JLab, would serve as an important check of our present day understanding of nuclear structure in terms of quark and gluon 
properties. Studies at $12 \mathrm{GeV}$ would provide important input for global analysis of observed spin and azimuthal asymmetries, leading to the separation of different contributions and extraction of underlying, essentially unexplored distribution and fragmentation functions.

\section{References}

1. J. P. Ralston, D. E. Soper, Nucl. Phys. B152 (1979) 109.

2. R. L. Jaffe, X. Ji, Nucl. Phys. B375 (1992) 527-560.

3. J. C. Collins, Nucl. Phys. B396 (1993) 161-182.

4. D. W. Sivers, Phys. Rev. D 41 (1990) 83.

5. P. J. Mulders, R. D. Tangerman, Nucl. Phys. B461 (1996) 197-237, erratumibid. B484 (1996) 538.

6. D. Boer, P. J. Mulders, Phys. Rev. D57 (1998) 5780-5786.

7. M. Anselmino and F. Murgia, Phys. Lett. B 442 (1998) 470.

8. S. J. Brodsky, D. S. Hwang, I. Schmidt, Phys. Lett. B530 (2002) 99-107.

9. V. Barone, A. Drago and P. G. Ratcliffe, Phys. Rept. 359,1 (2002).

10. J. C. Collins, Phys. Lett. B536 (2002) 43-48.

11. X. Ji, F. Yuan, Phys. Lett. B543 (2002) 66-72.

12. A. V. Belitsky, X. Ji, F. Yuan, Nucl. Phys. B656 (2003) 165-198.

13. J. Collins and A. Metz, Phys.Rev.Lett. 93, 252001 (2004).

14. A. Metz, M. Schlegel, Eur. Phys. J. A22 (2004) 489-494.

15. K. Goeke, A. Metz and M. Schlegel, Phys. Lett. B612, 233 (2005).

16. X. Ji, J.-P. Ma, F. Yuan, Phys. Rev. D71 (2005) 034005.

17. X. Ji, J.-P. Ma, F. Yuan, Phys. Lett. B597 (2004) 299-308.

18. H. Avakian, et al., Phys. Rev. D69 (2004) 112004.

19. H. Avakian, P. Bosted, V.Burkert and L. Elouadrhiri, nucl-ex/0509032.

20. K. Heller et al. 'Proceedings of Spin 96',Amsterdam,Sep.1996,p23

21. E704 collaboration (A. Bravar et al.), Phys.Rev.Lett. 77, 2626 (1996).

22. A. Airapetyan et al., Phys.Rev.Lett. 84, 4047 (2000).

23. A. Airapetyan et al., Phys.Rev. D55, 097101 (2001).

24. A. Airapetyan et al., Phys.Rev.Lett. D64, 097101 (2004).

25. V.Yu. Alexakhin et al.,Phys.Rev.Lett. 94, 202002 (2005).

26. Belle Collaboration (K. Abe et al.), hep-ex/0507063.

27. A. Efremov Annalen Phys. 13, 651 (2004)

28. A.M. Kotzinian and P.J. Mulders, Phys. Rev. D54 1229 (1996).

29. A. Kotzinian, Nucl. Phys. B 441, 234 (1995).

30. R.D. Tangerman and P.J. Mulders, Phys.Rev. D51 3357 (1995).

31. A. V. Efremov et al. Phys. Rev. D 67114014 (2003); hep-ph/0412420.

32. D.Boer and P. Mulders, Phys.Rev. D57, 5780 (1998).

33. J. Levelt and P. J. Mulders, Phys. Lett. B 338 (1994) 357 [hep-ph/9408257].

34. A. Bacchetta, P. J. Mulders, F. Pijlman, Phys. Lett. B595 (2004) 309-317.

35. F. Yuan, Phys. Lett. B 589, 28 (2004).

36. A. Afanasev and C. Carlson,hep-ph/0308163.

37. E. Avetisyan, A. Rostomyan, A. Ivanilov, hep-ex/0408002. 